\title{
Two-dimensional cadmium selenide electronic and optical properties: first principles studies
}

\author{
J M GALICIA-HERNÁNDEZ ${ }^{1, *}$, A SÁNCHEZ-CASTILLO ${ }^{2}$, L MORALES DE LA GARZA ${ }^{3}$ and \\ GREGORIO H COCOLETZI ${ }^{1}$ \\ ${ }^{1}$ Instituto de Física 'Luis Rivera Terrazas', Benemérita Universidad Autónoma de Puebla, Apartado Postal J-48, \\ Pue 72570, Mexico \\ ${ }^{2}$ Escuela Superior de Apan, Universidad Autónoma del Estado de Hidalgo, Chimalpa Tlalayote, Apan, Hidalgo, Mexico \\ ${ }^{3}$ Centro de Nanociencias y Nanotecnología, Universidad Nacional Autónoma de México, Ensenada, BC, Mexico \\ *Author for correspondence (josemariogahe@gmail.com)
}

MS received 18 July 2016; accepted 27 February 2017; published online 12 September 2017

\begin{abstract}
Structural, electronic and optical properties of two-dimensional (2D) cadmium selenide (CdSe) structures with $2 \times 2$ periodicities are investigated. First principles total energy calculations are performed within the periodic density functional theory. Initially, the structural properties are determined using the local density approximation as implemented in the PWscf code of quantum ESPRESSO package. To investigate the electronic properties, the GW method is applied to determine the energy gap within the plasmon pole and the random phase approximations. Optical properties are investigated to determine the dielectric constant and the Bethe-Salpeter theory is used to calculate the exciton binding energies. Zinc blende and wurtzite phases are considered to calculate the bulk energy gaps, which are compared to the experimental values, finding good agreement. The 2D structure exhibits an energy gap larger than that of the bulk, indicating the effects of reduction in dimensionality; these changes can be attributed to the dangling bonds that are present in the $2 \mathrm{D}$ layer.
\end{abstract}

Keywords. Cadmium selenide; optical properties; GW approach; Bethe-Salpeter approach.

\section{Introduction}

Cadmium selenide (CdSe) is a semiconductor compound of the II-VI family; it crystallizes in two different phases: zinc blende and wurtzite [1]. The experimental energy gap in the zinc-blende phase is $1.68 \mathrm{eV}$ [2] and in the wurtzite phase it is $1.74 \mathrm{eV}$ [3]. CdSe is transparent in the infrared regime of the electromagnetic radiation [4]. It has a variety of technological applications; it has been used in light-emitting diodes [5-8], solar cells, gamma ray detectors, photodetectors and optoelectronic [9-11] and photovoltaic devices [12-15]. The interest in CdSe is not only as a macroscopic material but also as a nanostructured material.

Low-dimensional nanostructures have been widely studied because of their extraordinary electrical and optical performances in comparison with the bulk counterpart. Recent experimental studies on the two-dimensional (2D) growth of CdSe nanocrystals have been reported that demonstrate the realization of $\mathrm{CdSe}$ nanosheets with lateral dimensions on the order of $700 \mathrm{~nm}$ and well-defined thickness [16]. Another study reported a free-standing nanosheet that was synthesized, with a thickness of $1.4 \mathrm{~nm}$ [17]. A theoretical work has been also published on 2D CdSe nanostructures studies to learn on the stability and possible applications in the fabrication of thin layer transistors [18]. Not only 2D-layers but also nanoparticles have been investigated to determine the best synthesis conditions and characterization $[19,20]$. In all cases, there are some open questions related to the change in the behaviour as a result of reduction in dimensionality.

After the discovery of graphene, nanosheets of binary compounds such as $\mathrm{CdSe}, \mathrm{CdS}, \mathrm{BN}, \mathrm{ZnO}$ and $\mathrm{AlN}$ have attracted the attention in nanoscience due to possible applications in technological fields. In general, 2D nanosheets having the same honeycomb lattice structure as that of graphene can be used to develop high- $T_{\mathrm{c}}$ superconductors [21], optical and semiconductor devices [22,23] and magnetic superlattices [24].

In CdSe nanosheets, the surface is polarized, which could lead to electrostatic and structural instability due to surface dangling bonds; these kinds of bonds are present because the $\mathrm{Cd}$ and $\mathrm{Se}$ atoms are unsaturated, unlike the four-coordinated atoms in bulk. The unsaturated atoms are considered active sites for adsorption of adatoms to form strong chemical bonds. It is possible to change the properties of $2 \mathrm{D}$ nanosheets by the saturation of dangling bonds; this can be done through partial or full hydrogenation. This technique allows the tuning of the gap, producing a change in the optical and magnetic properties of the nanostructure. Hydrogenation also decreases the internal stress between the atoms, making the nanosheet structurally very stable. Some theoretical results have been reported for clean CdSe nanosheets [25], but there are no reports for the hydrogenated one. In this work we 
made a comparative study between clean and hydrogenated $2 \mathrm{D} \mathrm{CdSe}-(2 \times 2)$ layers, observing crucial changes after hydrogenation; this study yields a good understanding of electronic, structural and optical properties before and after saturation.

\section{Model and method}

First principles total energy calculations have been performed to investigate the structural, electronic and optical properties of $2 \mathrm{D}$ cadmium selenide $(\mathrm{CdSe})$ nanostructures with $2 \times 2$ periodicities $(2 \mathrm{D} \mathrm{CdSe}-(2 \times 2))$. The present studies start by determining the ground state bulk lattice parameters of $\mathrm{CdSe}$ in the two most stable phases: cubic zinc blende $(6.08 \AA)$ and hexagonal wurtzite ( $a=b=4.28 \AA$ and $c=6.98 \AA$ ). Structural stabilizations have been investigated within the standard periodic density functional theory (DFT) as implemented in the PWscf code of the Quantum ESPRESSO package [26]. In the 2D geometries, two structures have been considered: a clean layer with unsaturated dangling bonds and a layer with both $\mathrm{Cd}$ and $\mathrm{Se}$ atoms saturated with hydrogen atoms. The $2 \mathrm{D} \mathrm{CdSe}-(2 \times 2)$ periodic nanostructure construction is done using the optimized bulk lattice parameters. For all cases (bulk and 2D) atoms and volume are allowed to relax (full geometry optimization) until the total force on each atom is smaller than $0.001 \mathrm{eV}^{-1}$. As stated earlier, studies are done using the DFT theory. In this work, electron-ion interactions are modelled with norm-conserving pseudopotentials, and the exchange-correlation energy is obtained using the local density approximation (LDA). Electron states are expanded in plane waves with energy cut of $50 \mathrm{Ry}$ and different $k$ points are invoked according to the Monkhorst-Pack scheme $(13 \times 13 \times 1$ for $2 \mathrm{D}$ systems and $13 \times 13 \times 13$ for the bulk case). To determine the lattice parameters in the zinc blende and wurtzite phases several pseudopotentials have been tested to choose those of pw that better reproduce the experimental results.

The $2 \mathrm{D} \mathrm{CdSe}-(2 \times 2)$ nanostructure in the hydrogen-free configuration has a lattice parameter $a=b=8.70 \AA$, and for the hydrogenated system $a=b=8.66 \AA$. Two adjacent 2D $\mathrm{CdSe}-(2 \times 2)$ layers (hydrogenated and non-hydrogenated) are separated by a vacuum gap of width $15 \AA$ in the perpendicular direction in order to avoid interaction between layers.

After atomic structure relaxations, electronic structure calculations have been carried out. As is well known, standard DFT underestimates the semiconductor energy gap; therefore, this parameter has been determined employing the $\mathrm{GW}$ approach. Within this GW theory, two approximations have been employed: the plasmon pole approximation (PPA) and the random phase approximation (RPA), as implemented in the Yambo project [27]. The band structures have been determined taking into account the high-symmetry sites within the first Brillouin zone. To complement the electronic structure studies the total density of states (DOS) and projected density of states (PDOS) have been explored.
Finally, optical properties have been investigated paying attention on the excitonic transitions. Both bulk and 2D layers are considered in the studies. As part of the optical properties characterizations the dielectric constant is determined taking into account excitonic transitions. Studies have been done within the Bethe-Salpeter formalism (BSE) [30] to determine the optical absorption.

\section{Results and discussion}

\subsection{Structural properties}

Bulk and 2D cadmium selenide nanosystems structural properties are first described. In the zinc blende phase, the calculated ground state lattice parameter is $6.06 \AA$, in good agreement with the experimental value of $6.08 \AA$, [28] while in the wurtzite phase it is found to be $a=b=4.28 \AA$ and $c=6.98 \AA$, also in agreement with experimental data; $a=b=4.30 \AA$ and $c=7.02 \AA$ [28]. The calculated bond length between $\mathrm{Cd}$ and Se atoms is $2.62 \AA$ in both phases, in good agreement with experimental value (2.61 $\AA$ ) [29]. Figure 1 shows the relaxed unit cell for both phases.

Two honey-comb-type (hexagonal) 2D CdSe- $(2 \times 2)$ structures are considered: non-hydrogenated and hydrogenated as mentioned in section 2. Hydrogen atoms are attached to $\mathrm{Cd}$ and $\mathrm{Se}$ atoms to saturate dangling bonds. In these configurations, the $\mathrm{H}$-atom interactions are reduced; however, the layer structure is not distorted drastically in comparison to the clean surface. Similar to the bulk studies, the lattice parameter is first determined using the DFT formalism within the LDA approach. This is followed by the energy gap calculation within the GW theory and finally the optical properties are explored using the Bethe-Salpeter approach [30] (next section). Two-dimensional $\mathrm{CdSe}-(2 \times 2)$ layer parameters have been determined in the hydrogen-free configuration $(a=b=$ $8.70 \AA$ ) and hydrogenated system ( $a=b=8.66 \AA$ ). Two adjacent 2D layers are separated by a vacuum gap of width $15 \AA$ in the perpendicular direction in order to avoid any interaction between layers. The $\mathrm{Cd}-\mathrm{Se}$ bond lengths are $2.53 \AA$ in the clean layer and $2.70 \AA$ in the hydrogenated structure. In the latter case, the corresponding $\mathrm{Cd}-\mathrm{H}$ and $\mathrm{Se}-\mathrm{H}$ bond lengths are 1.69 and $1.48 \AA$, respectively. These small bond lengths suggest the formation of $\mathrm{Cd}-\mathrm{H}$ and $\mathrm{Se}-\mathrm{H}$ strong bonds. In both cases, we observe a buckled structure after relaxation; the buckled heights are 0.34 and $1.02 \AA$ for clean and hydrogenated layer, respectively. The buckled heights are measured between $\mathrm{Cd}$ and Se atoms for both systems. Although hydrogenation leads to smaller lattice constant and larger buckled height, the hexagonal-like configuration is preserved. Figure 2 shows the relaxed structures.

\subsection{Electronic properties}

We have first investigated the ground state electronic properties within the standard DFT approach for CdSe in the wurtzite 
(a)

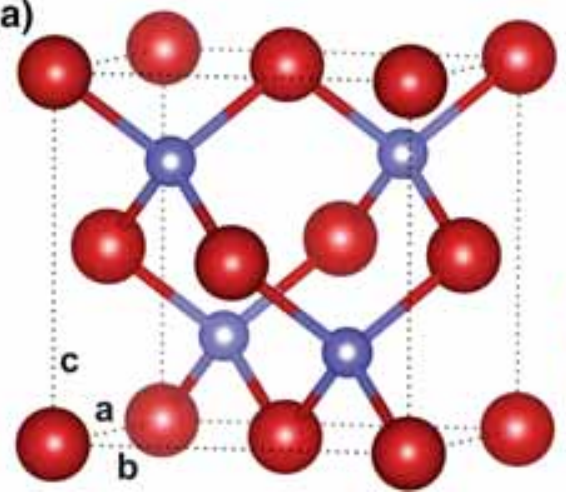

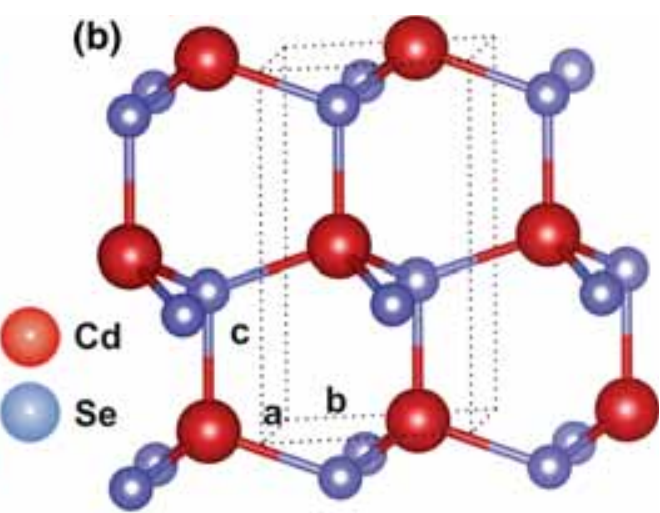

Figure 1. Relaxed unit cell for the bulk CdSe: (a) zinc-blende phase and (b) wurtzite phase.

(a)

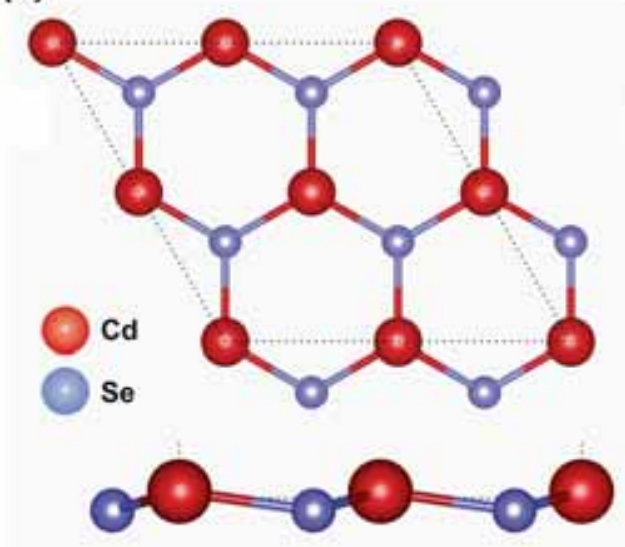

(b)

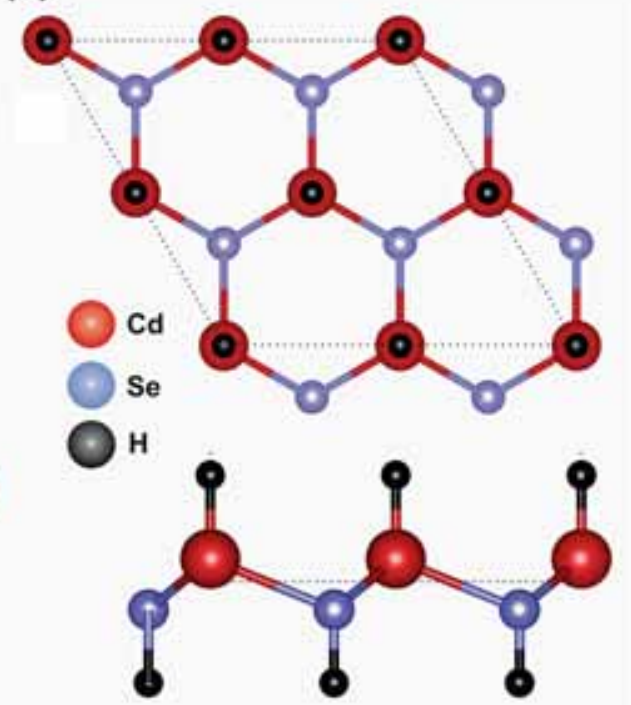

Figure 2. Relaxed structures of $2 \mathrm{D}-\mathrm{CdSe}-(2 \times 2)$ are shown for (a) non-hydrogenated and (b) hydrogenated cases.

and zinc blende phases and 2D CdSe- $(2 \times 2)$ layers (clean and hydrogenated). Wurtzite electronic structure shows a direct gap transition at the $\Gamma$ point with a value of $0.37 \mathrm{eV}$, far from the experimental value. This failure is because excited states are not considered within the standard DFT. For the zinc blende phase the corresponding direct energy gap is $0.29 \mathrm{eV}$ (see figure 3).

We now proceed to analyse the results obtained within the GW approach to determine the band gap using the LDA approximation. The GW method [31] is currently the most accurate first-principle approach for electronic band structure studies that gives the fundamental gaps of materials in good agreement with the experimental values. On the other hand, BSE calculations require as a starting point the quasiparticle band structure, usually obtained within the GW approximation.
The GW theory allows calculating properties of systems in excited states, beyond the standard DFT which only describes ground state properties. This theory is able to describe the propagation of an extra electron or one missing electron when direct or inverse photoemission occurs as a result of an excitation; this process generates a quasiparticle, which is an electron surrounded by the screening cloud.

In the GW approach the exchange-correlation potential considered by LDA of GGA theories is replaced by the selfenergy exchange-correlation operator, which is non-local, non-Hermitian, short range, complex and frequency dependent; it is a convolution of the Green's function $G$ and the screened Coulomb interaction $W$. The self-energy describes the interaction between electrons in a polarizable medium in a process involving an extra or missing electron. On one hand, $W$ describes the interaction between quasiparticles and it can 

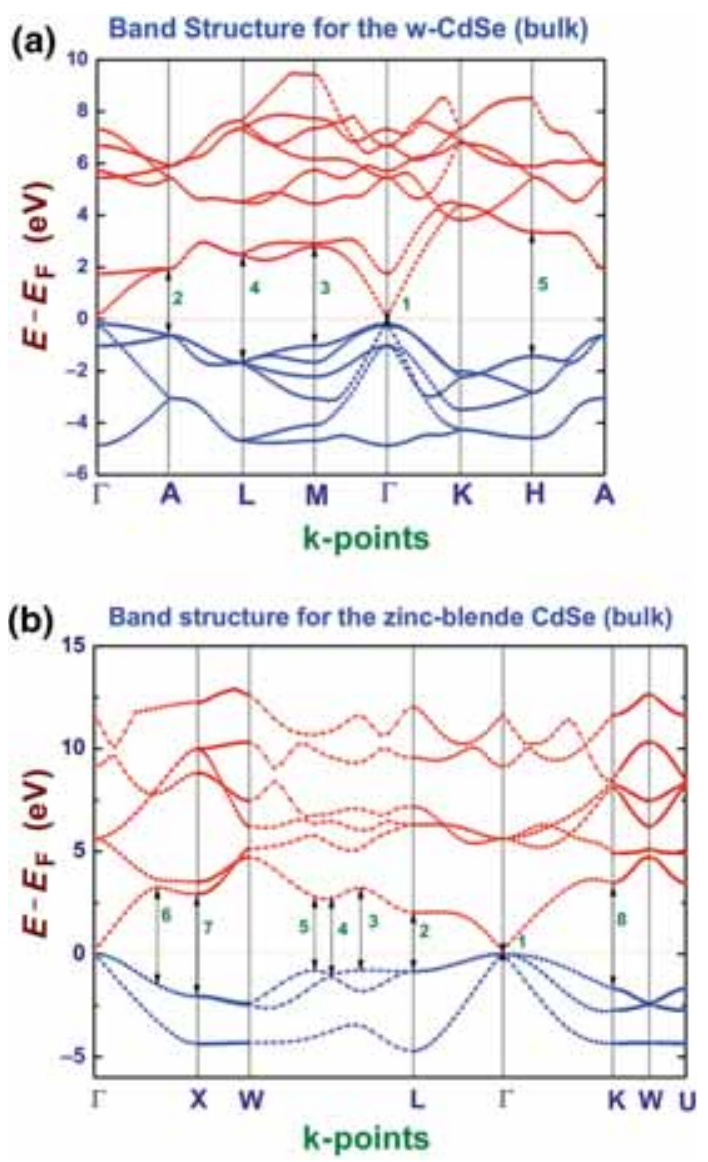

Figure 3. Electronic band structure for the (a) wurtzite and (b) zinc-blende CdSe (bulk system).

be understood as a screened potential, which is frequency dependent and weaker than the bare Coulomb interaction. On the other hand, Green's function contains spectral information on single-particle excitations.

In summary, the GW approach works as follows; The Kohn-Sham energies obtained by DFT are improved by the so called quasiparticle energies. Quasiparticle energies are obtained from the poles of the one-particle interacting Green's function; these energies correspond to the exact excitation energies.

By applying the GW approach we have improved the band gap value as compared with the standard DFT result; in the zinc blende phase the energy gaps is $1.61 \mathrm{eV}$ in the RPA and 1.68 in PPA with experimental value in the range of 1.68 $1.9 \mathrm{eV}$ [2]. In the wurtzite phase, the calculated energy gap value is $1.85 \mathrm{eV}$ in the RPA and $2.01 \mathrm{eV}$ in PPA, while experimentally the value is $1.74 \mathrm{eV}$ [3]. Results indicate a good agreement with the experimental data.

Electronic structure calculations are extended to $2 \mathrm{D} \mathrm{CdSe}-$ $(2 \times 2)$ structures; within the standard DFT approach the clean layer displays a band gap of $1.22 \mathrm{eV}$ (figure 4), whereas the hydrogenated structure shows an energy gap of $2.59 \mathrm{eV}$ (figure 6); results are summarized in table 1 .

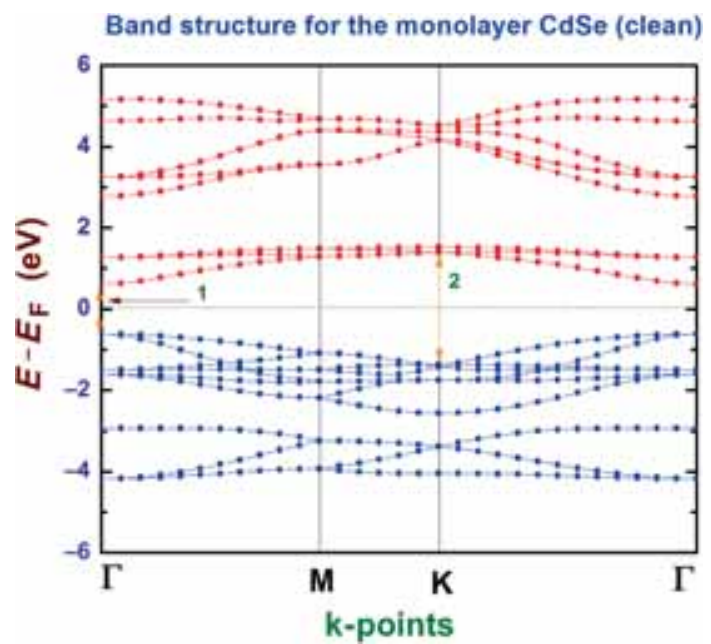

Figure 4. Electronic band structure for clean 2D CdSe.

Table 1. Obtained values for the band gap for all systems within different approximations.

\begin{tabular}{lcccc}
\hline & \multicolumn{4}{c}{ Band gap (eV) } \\
\cline { 2 - 4 } & & \multicolumn{2}{c}{ GW } & \\
\cline { 2 - 4 } Atomic structure & LDA & RPA & PPA & Exp. \\
\hline Zinc blende & 0.29 & 1.68 & 1.61 & $1.68-1.9[2]$ \\
Wurtzite & 0.37 & 1.85 & 2.01 & $1.74[3]$ \\
2D CdSe (clean) & 1.22 & 2.71 & 2.78 & - \\
2D CdSe (hydrogenated) & 2.59 & 4.77 & 4.79 & - \\
\hline
\end{tabular}

Figure 5 shows the PDOS of Cd and Se p-orbitals in the 2D $\mathrm{CdSe}-(2 \times 2)$ layer. We note that the main contribution slightly above the Fermi level comes from $\mathrm{p}_{z}$ orbitals; these orbitals form the surface dangling bonds generating the polarization at the surface, which may lead to electrostatic instability. Hydrogen atoms saturate dangling bonds, which in turn stabilize polar surfaces; in this way, $\mathrm{H}$ atoms act as an $\mathrm{n}$-doping source by donating electrons to saturate the dangling bonds. A change is observed in the hydrogenated $2 \mathrm{D}-\mathrm{CdSe}-(2 \times 2)$ band structure (figure 6 ) in comparison with the clean case as a result of surface states removal.

Let us consider the $2 \mathrm{D} \mathrm{CdSe}-(2 \times 2)$ non-hydrogenated and hydrogenated structures to determine the energy gap at the $\Gamma$ point by applying the GW theory. In the former case the band gap is $2.71 \mathrm{eV}$ in the RPA and $2.78 \mathrm{eV}$ in PPA, while for the latter structure it is $4.77 \mathrm{eV}$ in the RPA and $4.79 \mathrm{eV}$ in PPA. Note that these values are much larger than those of CdSe in the bulk.

A band gap enlargement is obtained by changing the dimensionality to a 2D structure; however, the electronic behaviour is not affected since the direct band gap characteristics at the $\Gamma$ point remain. This effect is also observed in the hydrogenated case, in which the band gap is even wider. 


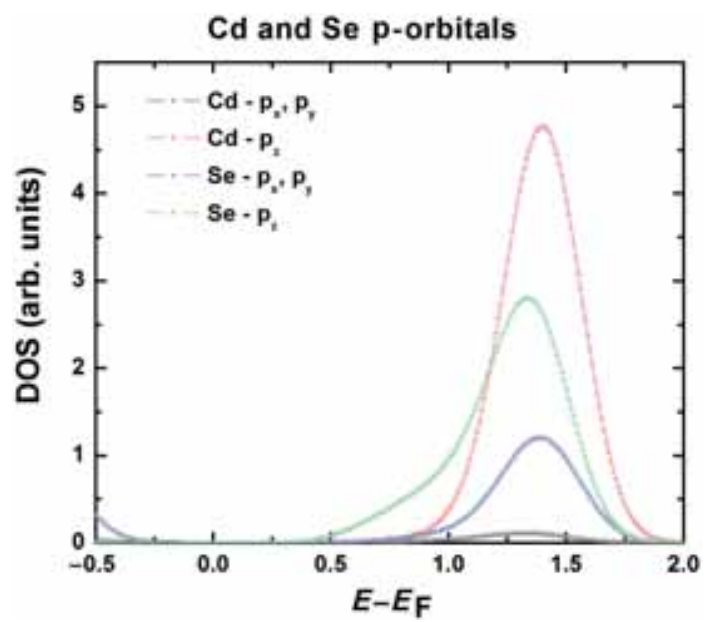

Figure 5. Projected density of states of p orbitals (clean 2D CdSe).

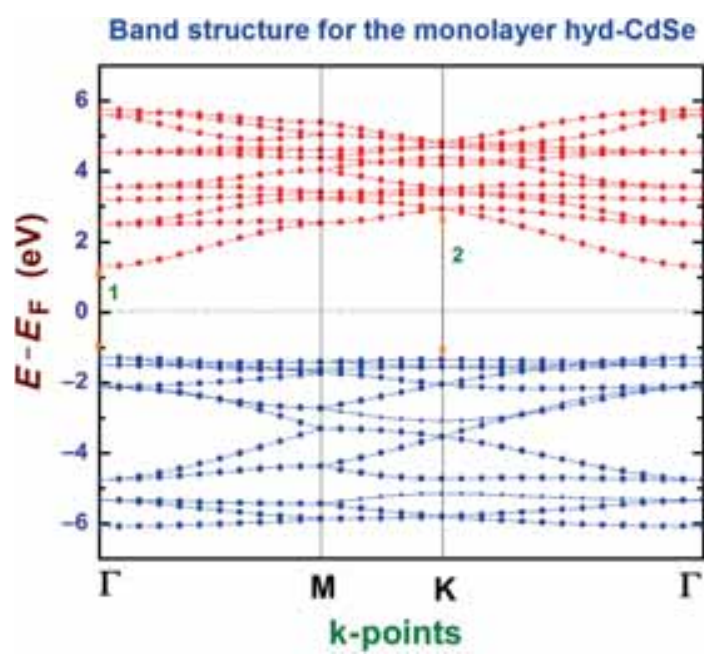

Figure 6. Electronic band structure for hydrogenated 2D CdSe.

\subsection{Optical properties}

Optical properties characterizations of materials are essential to determine technological applications. An important optical parameter is the dielectric constant, which may be calculated using classical or quantum mechanical theories.

Optical properties of semiconductors in the infrared, visible or ultraviolet spectrum are determined by the interband electronic transitions, which are also involved in the optical absorption. The dielectric function $\varepsilon(\omega)=\varepsilon_{1}(\omega)+i \varepsilon_{2}(\omega)$ describes the optical response of the material as a function of the photon energy. $E(\omega)$ and $\varepsilon_{2}(\omega)$ are related to the optical absorption. In this way, the imaginary part of the dielectric function can be used to describe electronic transitions, since it is related to the DOS of the valence and conduction bands.

The initial state of the systems (bulk and 2D) is the ground state. In this work, we deal with semiconductor structures; therefore, the ground state contains completely filled bands (valence) as well as completely empty bands (conduction); consequently, only interband transitions are considered in the analysis.

Peaks in the imaginary part of the dielectric function are due to direct band-band electronic transitions present in the band structure; also, drops in the intensity are related to the lack of bands with energy differences in the energy region in which the drop occurs.

In this report the dielectric constant is calculated using the Bethe-Salpeter equation (BSE) [30], which is a theory beyond the standard DFT formalism. To be precise, the BSE [30] theory is applied to calculate the optical absorption and then the imaginary part of dielectric constant. This Bethe-Salpeter formalism gives results in excellent agreement with experimental data. The electron-hole bound states (excitons) formation is an important physical phenomenon that is accounted for in the BSE theory. To make it clear, excitons are electronhole pairs that form states bound by their mutual screened Coulomb attraction. In the absorption spectrum yielded by the BSE method, excitonic peaks are obtained in the visible energy range. From these peaks, exciton binding energies are estimated; these energies are in the meV regime, which indicate weak electron-hole interactions. It is worthwhile mentioning that exciton binding energies are small in the bulk; this is incremented in confined systems such as quantum wells, quantum dots and 2D layers. The BSE approach allows obtaining the imaginary part of the dielectric function related to the absorption spectra and the peaks are connected with the spectral function obtained within standard DFT + the GW correction.

Figure 7 depicts the imaginary part of the dielectric function calculated using different approximations for both wurtzite and zinc blende phases. The imaginary part of the dielectric function is related to the electronic band structure since energy differences in the latter can be connected to peaks in the former, and these are associated with interband transitions.

As is well known, standard DFT yields only qualitatively good results; however, quantitatively this theory fails. Recall that the PDOS may be used to describe the orbitals that contribute to the electronic transitions. Moreover, when the energy shift generated by the GW correction is accounted for, the PDOS provides appropriate information concerning the electronic transitions. The electronic band structures together with the DOS are used to describe electronic transitions, which in turn indicate the exciton formation.

In figures 3, 4 and 6, we have labelled the electronic transitions related to the peaks in the imaginary part of the dielectric function of figures 7, 10 and 12 (bulk and 2D) with numbers 1,2, 3 and so on. The calculation of the imaginary part of the dielectric function has taken into account the energy shift induced by the GW approach. To complete the study, the PDOS was used to visualize the orbitals involved in each transition.

Figure 8 depicts the (wurtzite) CdSe PDOS. DOS calculations have been done to properly describe the electron transitions and the subsequent exciton formation, represented 


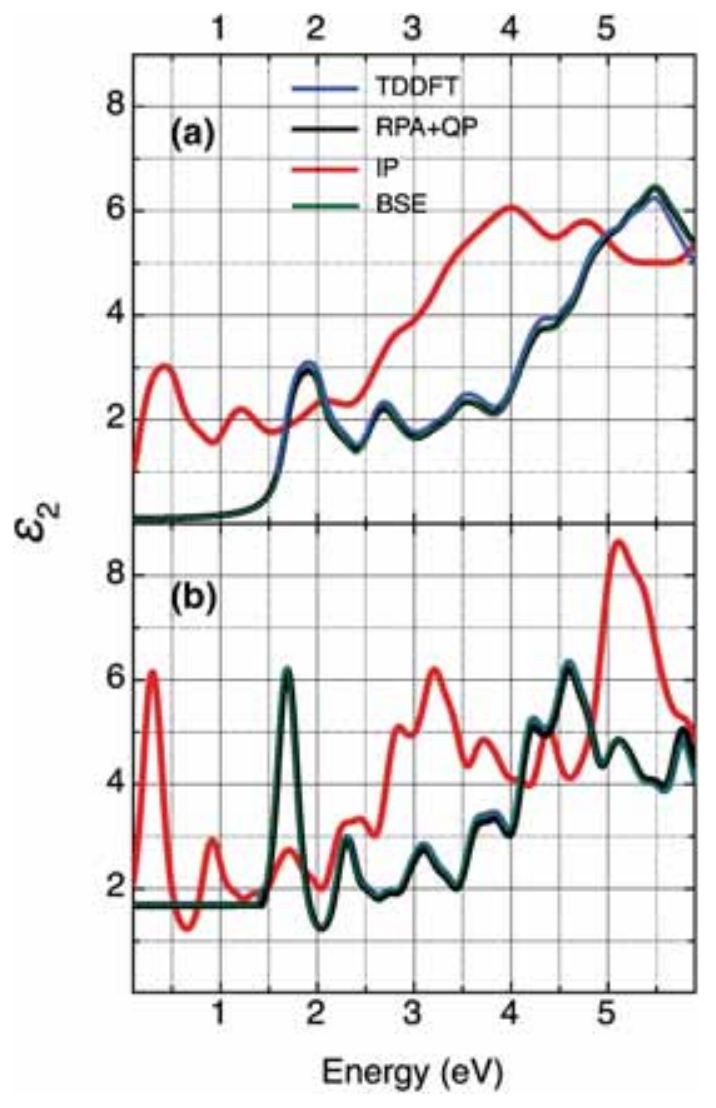

Figure 7. Imaginary part of the dielectric function within different approximations: (a) wurtzite phase and (b) zinc-blende phase.

in the dielectric function peaks. The DOS features may be described as follows: at energies below the Fermi level the most important contribution to the DOS is given by Cd-(p) and $-(\mathrm{s})$, and $\mathrm{Se}-(\mathrm{p})$ orbitals. At energies above the Fermi energy the Se-(d), Se-(p) and Cd-(p) make the most significant contributions to the DOS. This graph may throw some light concerning the possible electronic transitions in the system. Additionally, note from figure 8 the possible electronic transition from the orbital Se-(p) to Cd-(s). This is also suggested by the energy difference of these orbitals with the transition manifested at the first peak at $1.85 \mathrm{eV}$ in the dielectric function, see figure 7 .

Figure 8 exhibits another possible electronic transition, which is manifested as the peak at higher energies of the dielectric function. In this way the second peak at $2.75 \mathrm{eV}$ corresponds to a transition from Cd-(p) to Se-(p) or Se-(s). At even higher energies the third peak at $3.6 \mathrm{eV}$ corresponds to a transition from $\mathrm{Cd}$-(d) to $\mathrm{Se}-(\mathrm{p})$ or $\mathrm{Se}-(\mathrm{s})$. The fourth peak at $4.4 \mathrm{eV}$ is due to the transition from Se-(d) to $\mathrm{Cd}-(\mathrm{s})$, and finally the fifth peak at $5.5 \mathrm{eV}$ is originated by the transition from $\mathrm{Cd}-(\mathrm{p})$ to $\mathrm{Se}-(\mathrm{d})$.

DOS calculations for the zinc blende phase have also been performed. Figure 9 shows PDOS results, which allow determining the electron transitions, manifested as peaks in the dielectric function. This PDOS is again useful to visualize
Projected DOS for the w-CdSe (bulk)

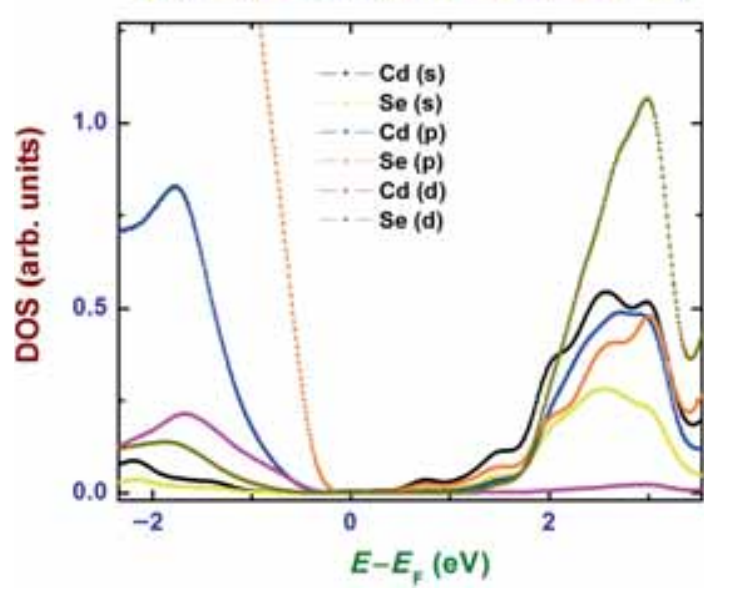

Figure 8. Projected density of states in an energy range of $6 \mathrm{eV}$.

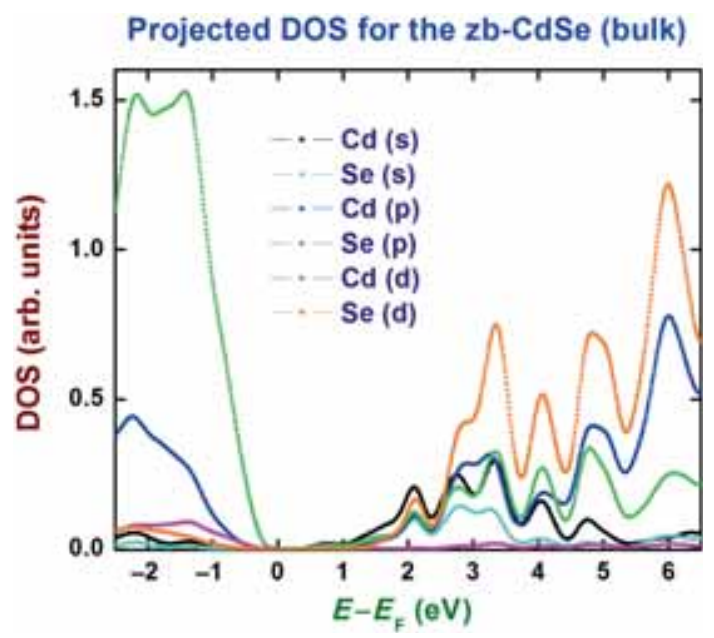

Figure 9. Projected density of states for bulk zb-CdSe.

the orbitals involved in the transitions. At $1.6 \mathrm{eV}$ the dielectric function exhibits a peak, which corresponds to an electron transition from the Se-(p) orbital to the Cd-(s) orbital. At $2.5 \mathrm{eV}$ a peak appears, which is associated with a transition from the Se-(p) orbital to the Se-(d) orbital.

In figure 9, at energies bellow the Fermi level, the main contribution to the DOS comes from Se-(p) and Cd-(p) orbitals. It may be argued that the peaks in the dielectric function at $3.1,3.8,4.25,4.75,5.1$ and $5.8 \mathrm{eV}$ are all related to electron transitions from the Se-(p) as well as Cd-(p) orbital to a Se(d) orbitals. At higher energies, there are peaks in the DOS, which originate from the Se-(d) orbitals.

In what follows, results of the 2D layer structures are described. In figures 4 and 6 , we have labelled the electronic transitions related to the peaks in the imaginary part of the dielectric function of figures 10 and 12 (2D layer) with numbers 1, 2, 3 and so on. Similar to the bulk cases, in calculations for the imaginary part of the dielectric function, the energy shift induced by the GW approach has been taken into 
Im $\varepsilon(\omega)$ of the clean CdSe nanosheet

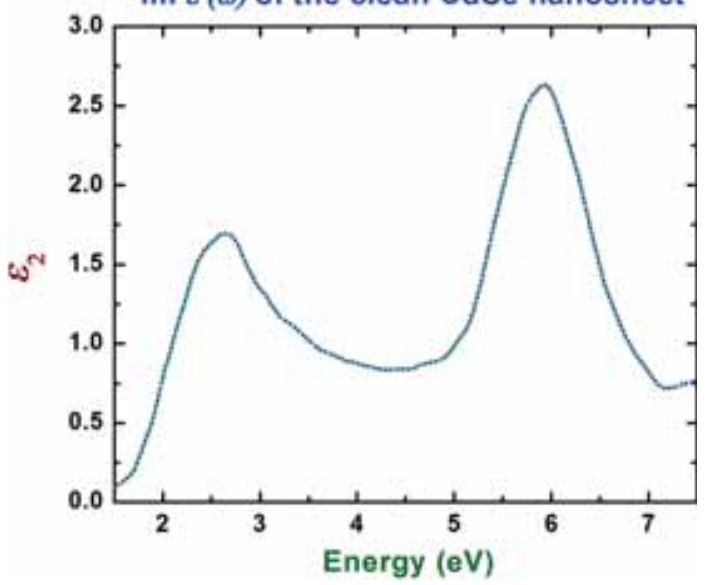

Figure 10. Imaginary part of the dielectric function for 2D CdSe (clean) is shown in the figure.

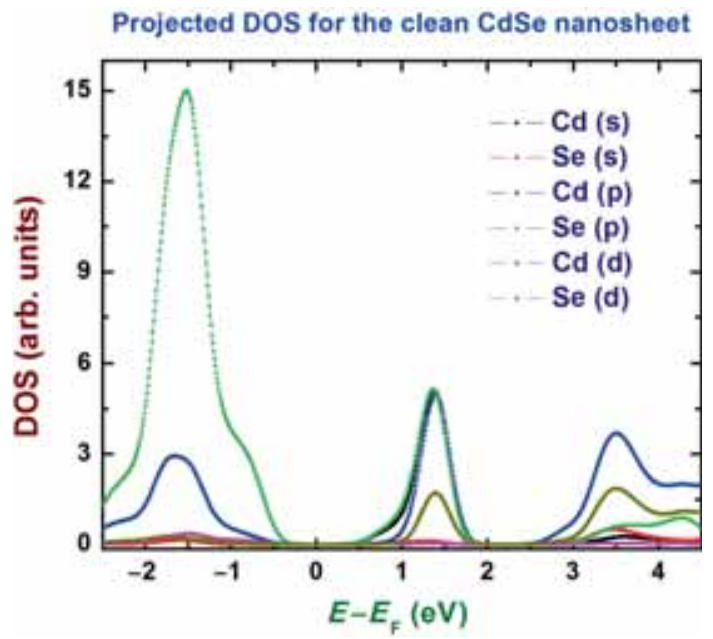

Figure 11. Projected density of states for 2D CdSe (clean).

account. To complete the study, the PDOS was used to visualize the orbitals involved in each transition.

Figure 10 shows the imaginary part of the dielectric function corresponding to the $2 \mathrm{D} \mathrm{CdSe}-(2 \times 2)$ layer $($ clean $)$ within the Bethe-Salpeter formalism. The graph displays two peaks, the first at $2.7 \mathrm{eV}$ and the second at $6 \mathrm{eV}$.

As before, it is possible to determine the orbitals involved in the electron transitions. In figure 10 the peak at $2.7 \mathrm{eV}$ corresponds to a transition of an electron from the Se-(p) to the Cd-(s) orbital (see figure 11). At $1.3 \mathrm{eV}$ an overlap between Cd-(s) and Cd-(p) orbitals is obtained; at this energy, the orbital heights are of the same order; therefore, the transition may be from Se-(p) orbital to Cd-(s) as well as to Cd-(p) orbital. The peak at $6 \mathrm{eV}$ indicates that the transition is from the Se-(p) to the Cd-(p) orbital.

$\mathrm{GW}$ calculation yielded a band gap equal to $4.7 \mathrm{eV}$ for the hydrogenated 2D layer. Consequently, the excitonic effects are expected to be weak because of the large band gap, and
$\operatorname{Im} \varepsilon(\omega)$ for the hyd-CdSe nanosheet

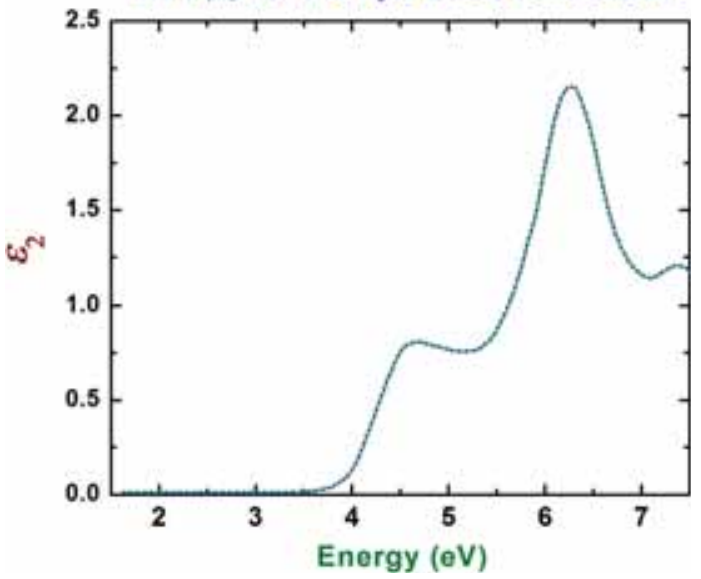

Figure 12. Imaginary part of the dielectric function for $2 \mathrm{D} \mathrm{CdSe}$ (hydrogenated).

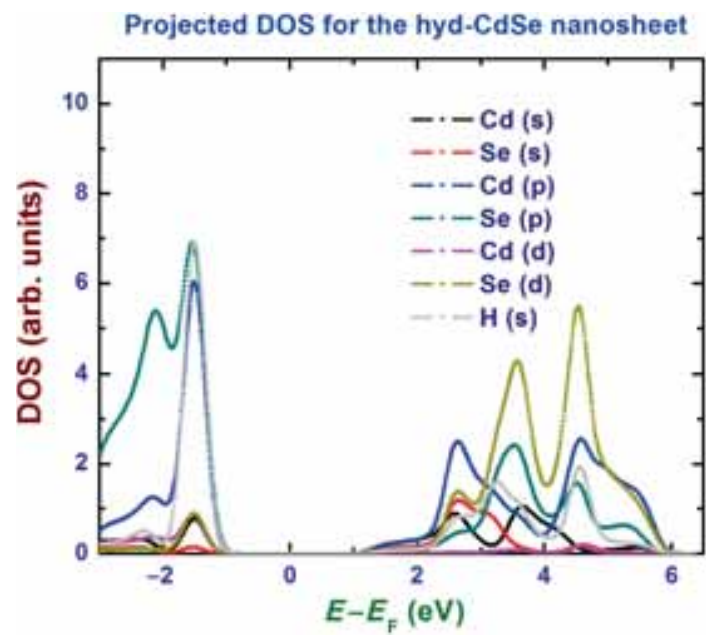

Figure 13. Projected DOS for hydrogenated 2D CdSe.

the use of the Bethe-Salpeter approximation may be unimportant. It shall be pointed out that optical properties within this approximation may be well described for values smaller than the band gap $(4.5 \mathrm{eV})$. In contrast, the time-dependent density functional theory (TDDFT) [32] is a good approach to determine the imaginary part of dielectric function, which gives information on the electronic transitions as BSE approach does.

Figure 12 depicts the dielectric function imaginary part for hydrogenated 2D CdSe within the TDDFT approach. Two peaks are obtained, the first at $4.7 \mathrm{eV}$, and the second at $6.3 \mathrm{eV}$. The first peak is produced by an electron transition from the $\mathrm{H}-(1 \mathrm{~s})$ to the Cd-(p) orbital, and the second peak is a result of the transition from $\mathrm{H}-(1 \mathrm{~s})$ to Se-(d) orbital (see figure 13).

Figure 13 shows the PDOS for the hydrogenated layer. The features indicate that the hydrogen 1s orbitals play an important role because below the Fermi level all the orbitals have a peak, i.e., there is full orbitals hybridization. The hydrogen 


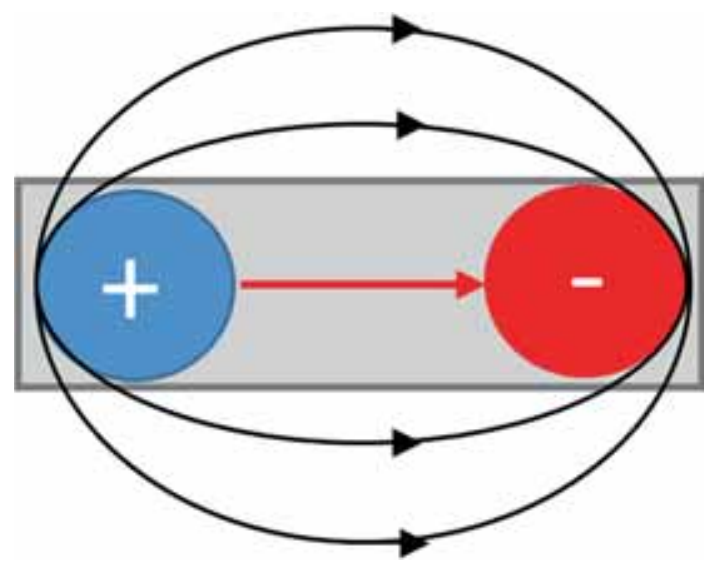

Figure 14. Potential lines of the electron-hole interaction.

orbitals generate saturated surface states, which induces the band gap opening. The figure shows a strong overlap of all $\mathrm{Cd}$ and Se orbitals with H-s orbitals (just below the Fermi level); this fact confirms the creation of a strong chemical bond between $\mathrm{H}$ and $\mathrm{Cd}-\mathrm{Se}$ atoms. From hydrogenation we can conclude that the gap opening is possible due to hydrogen electrons filling up the original empty lowest orbitals of conduction band in the clean 2D nanostructure.

3.3a Exciton binding energies: An important parameter to be determined is the exciton binding energy, which can be calculated as follows:

$$
E_{\text {binding }}=E_{\mathrm{BSE}}-E_{\mathrm{GW}},
$$

where $E_{\text {binding }}$ is the exciton binding energy, $E_{\mathrm{BSE}}$ is the energy at which the first peak is observed in the absorption spectrum in the BSE approach and $E_{\mathrm{GW}}$ is the corresponding energy at which the first peak in the GW approach is obtained.

The wurtzite exciton binding energy is $17 \mathrm{meV}$ within the BSE calculations. On the other hand, the $2 \mathrm{D} \mathrm{CdSe}-(2 \times 2)$ non-hydrogenated exciton binding energy is $58 \mathrm{meV}$, which is roughly four times the value for bulk CdSe (wurtzite). This bulk exciton binding energy is in good agreement with the experimental value $(15 \mathrm{meV})$ [33]. On the other hand, it is known that the effective mass theory yields, for the 2D structure ground state exciton energy, four times that of the bulk [34]. Note that results resemble those corresponding to the effective mass theory. For the bulk CdSe (zinc blende) the calculated exciton binding energy is $(11 \mathrm{meV})$ also in good agreement with the experimental value $(15 \mathrm{meV})$ [33].

The increase in the exciton binding energy in the $2 \mathrm{D}$ CdSe in comparison with the bulk system can be attributed to the reduction in the screening effects; this leads to a stronger attraction between electrons and holes; in this way, the binding energy necessary to decouple them is larger. A schematic of this fact is shown in figure 14 .

The figure depicts the many Coulomb potential lines staying out of the system plane which correspond to the 'unscreened interaction' (black curves), while the in-plane (red line) represents the screened interaction. This allows us to understand that when the dimensionality is reduced from $3 \mathrm{D}$ (bulk) to 2D, the screening is weaker in the latter case and the electron-hole interaction is stronger.

When the 2D CdSe is hydrogenated, the dangling bonds are saturated; the presence of the hydrogen atoms leads to an increase of the screening in comparison with the clean structure and the electron-hole attraction is consequentially reduced, leading to a smaller exciton binding energy; therefore, we can assume that the excitonic effects will be less important for the hydrogenated system; hence, the study of excitonic effects for the hydrogenated case is not included in this work.

\section{Conclusions}

Wurtzite and zinc-blende bulk CdSe structural, electronic and optical properties have been investigated and the results are in good agreement with experiments. Studies are extended to $2 \mathrm{D}$-CdSe- $(2 \times 2)$ layers (hexagonal arrangement), with two cases being considered, the clean layer and the hydrogenated structure where hydrogen atoms were added to the surface. The electronic behaviour is not modified by changing the dimensionality, since the 2D structures, in both cases, present a direct band gap at the gamma point; nevertheless, the band gap is opened with the reduction of dimensionality and it is even larger for the hydrogenated case as produced by the surface states modification. Band gap calculations within the standard DFT have been improved using the GW approach. Bulk results are in good agreement with experiments. However, in the 2D structures, no comparison with experimental data has been done as the 2D structures have not been investigated experimentally. The bulk and clean 2D layer imaginary parts of the dielectric function were computed using the optical absorption spectra within the Bethe-Salpeter approach. Results provide information on the optical properties and the excitonic binding energies, with the findings in good agreement with experiments. For the hydrogenated 2D structure, the excitonic effects are weaker because of the large band gap; hence the Bethe-Salpeter approach is unnecessary in this case; nevertheless, the imaginary part of the dielectric function was computed within the TDDFT approach.

\section{Acknowledgements}

GHC acknowledges the financial support of VIEP-BUAP, grant 31/EXC/06-G, CONACYT Project \#223180 and Cuerpo Académico Física Computacional de la Materia Condensada (BUAP-CA-191). Calculations were performed in the DGCTIC-UNAM Supercomputing Center. Calculations have been also performed at the 'Laboratorio Nacional de Supercomputo del Sureste', BUAP. 


\section{References}

[1] Datta S, Saha-Dasgupta T and Sarma D D 2008 J. Phys.: Condens. Matter 20445217

[2] Gutowski J, Sebald K and Voss T 2009 Semiconductors, vol. 44B (Berlin: Springer) p 75

[3] Madelung O 2004 Semiconductors data handbook (Berlin: Springer) p 815

[4] Efros A L and Rosen M 2000 Annu. Rev. Mater. Sci. 30475

[5] Colvin V L, Schlamp M C and Alivisatos A P 1994 Nature 370 354

[6] Schlamp M C, Peng X and Alivisatos A P 1997 J. Appl. Phys. 825837

[7] Nirmal M, Dabbousi B O, Bawendi M G, Macklin J J, Trautman J K, Harris T D et al 1996 Nature 383802

[8] Schreuder M A, Xiao K, Ivanov I N, Weiss S M and Rosenthal S J 2010 Nano Lett. 10573

[9] Jie J, Zhang W, Jiang Y and Lee S 2006 Appl. Phys. Lett. 89 133118

[10] Qu L and Peng X 2002 J. Am. Chem. Soc. 1242049

[11] Frese K W 1982 Appl. Phys. Lett. 40275

[12] Sun B and Greenham N C 2006 Phys. Chem. Chem. Phys. 8 3557

[13] Hamilton C E, Flood D J and Barron A R 2013 Phys. Chem. Chem. Phys. 153930

[14] Amin S A, Salim S T, Salam K M A and Galib M A A 2013 Int. J. Electr. Energy 11

[15] Sun B, Marx E and Greenham N C 2003 Nano Lett. 3961

[16] Bouet C, Mahler B, Nadal B, Abecassis B, Tessier M D, Ithurria S et al 2013 Chem. Mater. 25639
[17] Son J S, Wen X D, Joo J, Chae J, Baek S I, Park K et al 2009 Angew. Chem. 1216993

[18] Wen X D, Hoffmann R and Ashcroft N W 2013 Adv. Mater. 25 261

[19] Hao E, Sun H, Zhou Z, Liu J, Yang B and Shen J 1999 Chem. Mater. 113096

[20] Lokteva I, Radychev N, Witt F, Borchert H, Parisi J and KolnyOlesiak J 2010 J. Phys. Chem. C 11412784

[21] Gamble F R and Silbernagel B G 1975 J. Chem. Phys. 632544

[22] Liu M, Yin X B, Avila E U, Geng B S, Zentgraf T, Lu L et al 2011 Nature 47464

[23] Lin Y M, Dimitrakopoulos C, Jenkins K A, Farmer D B, Chiu H Y, Grill A et al 2010 Science 327662

[24] Zhou J, Wang Q, Sun Q, Chen X S, Kawazoe Y and Jena P 2009 Nano Lett. 93867

[25] Yu G, Chen N, Chen L, Xie Y, Wang F and Ye X 2014 Phys. Status Solidi A 211952

[26] Giannozzi P, Baroni S, Bonini N, Calandra M, Car R, Cavazzoni C et al 2009 J. Phys.: Condens. Matter 21395502

[27] Marini A, Hogan C, Grüning M and Varsano D 2009 Comput. Phys. Commun. 1801392

[28] Tomasulo A and Ramakrishna M V 1996 J. Chem. Phys. 105 3612

[29] Hadjipanayis G C and Siegel W 1994 Nanophase materials: synthesis-properties-applications (USA: Springer) p 475

[30] Salpeter E E and Bethe H A 1951 Phys. Rev. 841232

[31] Hedin L 1965 Phys. Rev. 139 A796

[32] Runge E and Gross E K U 1984 Phys. Rev. Lett. 52997

[33] Scholes G D and Rumbles G 2006 Nat. Mater. 5683

[34] Tran-Thoai D B 1990 Physica B: Condens. Matter 164295 